\title{
Simulación dinámica del impacto de la gestión de riesgo en empresas de transporte
}

\author{
Práxedes Antonio Torres Ortega 1,2,3* \\ Práxedes Antonio Torres Ortega \\ ${ }^{1}$ Universidad Tecnológica de Panamá, Facultad de Ingeniería Industrial, Panamá, República de \\ Panamá \\ ${ }^{2}$ Universidad del Istmo, Facultad de Negocios, Panamá, República de Panamá \\ ${ }^{3}$ Universidad Católica Santa María la Antigua, Facultad de Negocios, Panamá, República de Panamá
}

*Autorpara correspondencia. E-mail: praxedes.torres@utp.ac.pa

Recibido: 12 de noviembre de 2019

Aceptado: 06 de diciembre de 2019

\begin{abstract}
Resumen
Actualmente, los indicadores mundiales en cuanto a fatalidades por accidente de tránsito están en alza y preocupan a nivel global. En Panamá, se tiene una tendencia de crecimiento en la aplicación de gestión de riesgo en seguridad vial, en los transportistas de combustible claro. En la medida que el nivel de gestión de riesgo vial se implementa y crece su aplicación, el transporte puede llegar a reducir hasta un $27 \%$ las fatalidades por accidentes de tránsito en la industria del transporte de combustible claro en Panamá.
\end{abstract}

Palabras clave: ISO 39001, Accidentes viales, fatalidades de viales, transportistas, simulación dinámica.

\begin{abstract}
Currently, global indicators regarding traffic accident fatalities are on the rise and are of global concern. In Panama, there is a growth trend in the application of risk management in road safety, in clear fuel carriers. To the extent that the level of road risk management is implemented and its application grows, transport can reduce traffic fatalities by up to $27 \%$ in the clear fuel transportation industry in Panama.

Keywords: ISO 39001, Road accidents, road fatalities, freigth carriers, dynamic simulation.

\section{Introducción}

La cantidad de accidentes de tránsito y las fatalidades como causas de ellos, son considerables a nivel mundial como un problema global (OMS, 2015). Así en el 2009, se realiza la Primera Conferencia Ministerial Mundial sobre Seguridad Vial, organizada por el Gobierno de la Federación de Rusia. Luego en marzo de 2010, se realizó La Asamblea General de las Naciones Unidas, la cual proclamó oficialmente el Decenio de Acción para la Seguridad Vial 2011-2020. El Decenio de Acción
\end{abstract}


(Organización Mundial de La Salud, 2016) busca el compromiso de las naciones firmantes para que hagan esfuerzos en reducir la cantidad de fatalidades producto de los accidentes viales; la meta acordada fue reducirla en una cantidad de accidentes al año 2020 a nivel nacional, regional y mundial (Sminkey, 2010). Este tipo de acuerdos de organizaciones mundiales como las Naciones Unidas ha hecho que el tema de seguridad vial tome relevancia y sea un tema por tratar en la agenda de las naciones.

Este documento tiene como primera intención mostrar el modelado de la influencia que tiene la aplicación de la gestión de riesgo vial por parte de las compañías de transporte para reducir la cantidad de accidentes viales y también las fatalidades productos de estos accidentes. Se tomará como marco de referencia la industria del transporte de combustible claro para describir, el nivel de gestión de riesgo y poder llegar a un modelo que diera la predictividad de accidentes y fatalidades tomando el nivel de Gestión de Riesgos como una de las principales variables y la herramienta de la dinámica de sistemas para simular (Sterman, 2000).

La variable dependiente del modelo es la cantidad de accidentes viales dadas por las variables independientes como la probabilidad de accidentabilidad dada el nivel de gestión de riesgos de la organización y el número de viajes realizados dada la demanda de combustible nacional.

El nivel de gestión de riesgos se mide de diversas formas en base al nivel de cumplimiento de la norma ISO 39001:2012 (International Standard Organization, 2017). El nivel de gestión de riesgo podrá ofrecer la probabilidad de accidentabilidad por viaje de las compañías de transporte. La otra variable que genera riesgos de accidentes es la cantidad de viajes realizados por parte de los transportistas, este valor es obtenida por parte de las encuestas a los transportistas. El número de viajes es influenciado por la demanda de combustible que se tiene en el país.

Al final se muestra el avance que se está teniendo en el país en cuanto a los accidentes y fatalidades viales.

\section{Materiales y métodos}

La presente investigación es del tipo predictivo y de diseño no experimental del tipo transversal. La investigación busca predecir el impacto de diferentes niveles de gestión de riesgo en el sector de transporte de combustible que puedan tener en la cantidad de accidentes de tránsito y por ende fatalidades como consecuencias de ellos.

Para medir el nivel de gestión de riesgo, se diseñó y aplicó una encuesta diseñada en base a la Norma ISO 39001:2012 para medir el nivel de cumplimiento alcanzado en gestión de seguridad vial.

Se tomaron muestras del sector del transporte de combustible a granel que brindan sus servicios en La República de Panamá. Se realizó una prueba piloto para calcular el tamaño de la muestra utilizando los siguientes valores para cada variable: 
$\mathrm{Z}=1.96$ (Nivel de confiabilidad de 95\%)

$\mathrm{e}=0.025$

Media $=0.95$ como nivel de cumplimiento con la norma.

$\mathrm{N}=27$ encuestas

Finalmente se aplicaron las encuestas a 37 compañías de transporte de combustible (Torres, 2017). Dado esta cantidad de encuestas, el error bajo de 0.025 a 0.021

La encuesta se aplicó a los administradores de operaciones de las empresas de transporte responsables de los temas de gestión de riesgo vial. Se les solicitó que se auto evaluaran el nivel de cumplimiento con la norma ISO 39001:2012 tanto para los años 2014, 2015 y 2016.

La encuesta evalúa el nivel de gestión de riesgo vial, tomando el nivel de cumplimiento de la norma ISO 39001:2012. Se tomó el promedio de respuestas dadas con la respuesta "SI", que indica que se cumple con el punto que la norma pide cumplir. Al final se contabilizan la cantidad de respuestas positivas del total de preguntas de la encuesta. Por ejemplo: si el encuestado respondió 54 preguntas con las respuestas "SI" de un total de 58 preguntas, relacionadas con la gestión de riesgo, el puntaje obtenido sería de 0.93 en general, es decir $93 \%$ de cumplimiento. Luego, se promedia estos índices para calcular el nivel alcanzado promedio entre todas las compañías de transporte.

Para las tabulaciones, cálculos de promedios, análisis y gráficas se utilizaron las herramientas SPSS versión 20; así como Microsoft Excel.

Se toman datos secundarios como la demanda de combustible claro en la República de Panamá dada en la Secretaría Nacional de Energía. Las variables de niveles de gestión de riesgos, cantidades de viajes, accidentes, fatalidades, son obtenidas de las encuestas. Luego se aplica una matriz de correlaciones entre las variables para determinar sus relaciones.

Para diseñar el modelo de predicción de fatalidades por accidentes de tránsito del sector de transporte de combustible, se simuló utilizando la Dinámica de Sistemas como modelado. Por motivos, de practicidad se asumió un comportamiento normal de los datos.

La demanda de producto transportado se basará en los registros públicos que se cuentan de demanda de combustible claro dados por La Secretaría Nacional de Energía medidas en Galones o Litros.

\section{Resultados}

\subsection{Encuestas}

Las encuestas fueron aplicadas a representantes de administradores de operaciones en las compañías de transporte, para que dieran sus percepciones sobres el cumplimiento de la norma ISO 39001:2012 para los años 2014 a 2016. Los resultados fueron los siguientes (Torres, 2017):

El promedio general del nivel de seguridad vial para el 2016 es de $91 \%$ para el sector de transporte de combustible claro, el cual se puede considerar alta y adicionalmente de tener un crecimiento considerable entre los años 2014 al 2016. A un nivel de confiabilidad de 95\%, la estimación del 
intervalo del nivel de seguridad vial promedio en el transporte de combustible claro se encuentra entre 88\% a 93\% el nivel de cumplimiento. Lo cual es muy buen resultado para esta industria. Adicionalmente se resalta una crecida considerable en el cumplimiento de la norma. En la Gráfica 1 se puede notar estos resultados.

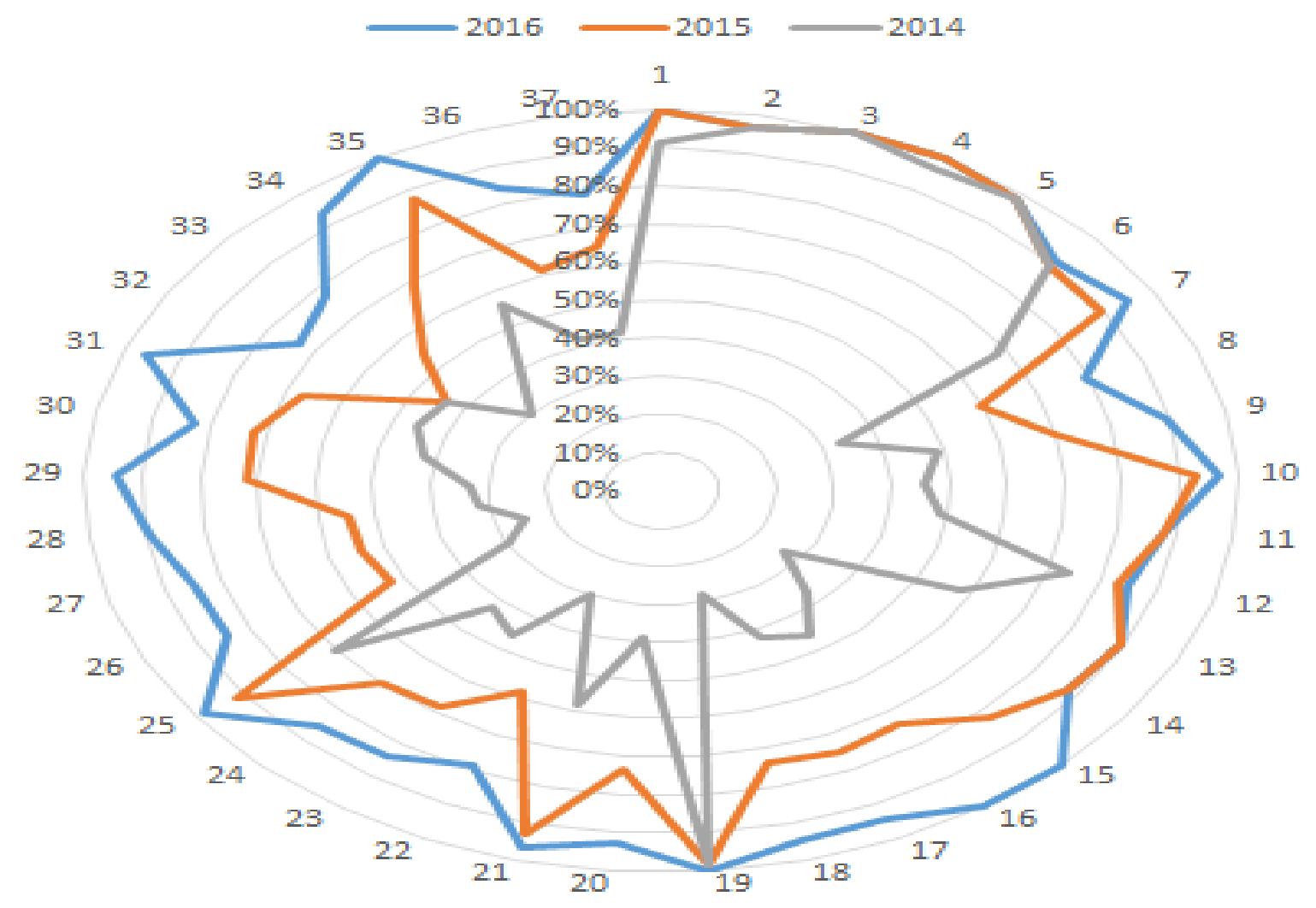

Gráfico 1: Nivel de Gestión de Riesgo en el Transporte de Combustible 2014-2016

En cuanto al nivel de cumplimiento por cada uno de los puntos de la norma, en promedio el nivel alcanzado por las 37 empresas en cada año fueron los mostrados en la Gráfica 2. 


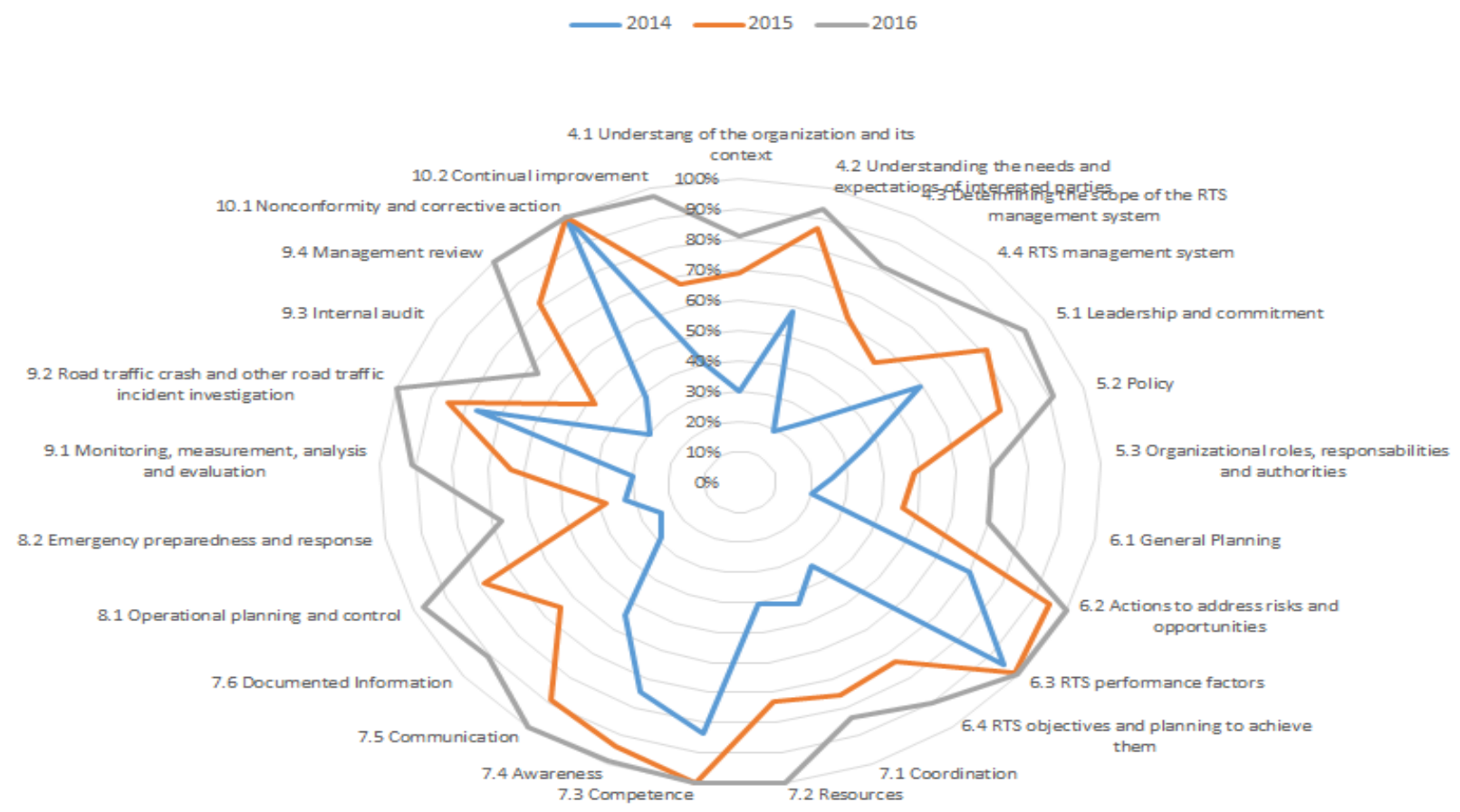

\section{Gráfico 2: Nivel de Gestión de Riesgo en el Transporte de Carga de Combustible por Elemento de la Norma ISO 39001:2012}

Aunque se cuenta con solo tres años de referencia, se hace la asunción de normalidad y se calcula una matriz de correlaciones bivariados de las siguientes variables: el nivel de gestión de riesgo alcanzado; la cantidad de accidentes viales tenidos por el transportista, así como las fatalidades productos de ellas; y cantidad de viajes. En la Tabla 1, se muestran las cantidades y las correlaciones respectivas de cada variable.

Tabla 1: Correlaciones de las principales variables de Accidentes de Tránsito

\begin{tabular}{|c|c|c|c|c|c|c|c|}
\hline Variables & $\begin{array}{l}\text { Número } \\
\text { Fatalidade } \\
\mathrm{s}\end{array}$ & $\begin{array}{l}\text { Númer } \\
\text { o } \\
\text { Herido } \\
\mathrm{s}\end{array}$ & $\begin{array}{l}\text { Número } \\
\text { Accident } \\
\text { es }\end{array}$ & $\begin{array}{l}\text { Número } \\
\text { Vehículo } \\
\text { s }\end{array}$ & $\begin{array}{l}\text { Número } \\
\text { Viajes }\end{array}$ & $\begin{array}{l}\text { Nivel } \\
\text { de } \\
\text { GR }\end{array}$ & $\begin{array}{l}\text { Legislaci } \\
\text { ón }\end{array}$ \\
\hline Número de & & & & & & & \\
\hline $\begin{array}{l}\text { Fatalidades } \\
\text { Número de }\end{array}$ & 1 & -0.797 & 0.997 & 0.216 & 0.978 & 0.388 & -0.155 \\
\hline $\begin{array}{l}\text { Accidentes } \\
\text { Número de }\end{array}$ & 0.997 & -0.841 & 1 & 0.290 & 0.991 & 0.457 & -0.229 \\
\hline Viajes & 0.978 & -0.906 & 0.991 & 0.416 & 1 & 0.573 & -0.358 \\
\hline Nivel de GR & 0.388 & -0.866 & 0.457 & 0.984 & 0.573 & 1 & -0.970 \\
\hline
\end{tabular}


Estos datos indican, que las correlaciones más importantes a utilizar para pronosticar los accidentes y fatalidades de tránsito, los cuales son:

El número de fatalidades (F) con el número de accidentes (0.997) y con el número de viajes (0.978) El número de accidentes tiene fuerte correlaciones (0.997) con el número de fatalidades y con el número de viajes (0.991). Con respecto al nivel de gestión de riesgo la correlación es positiva, pero baja con 0.457 Dada esta consideración, NO se tomará en el modelo de simulación, la correlación del número de accidentes con el nivel de gestión de riesgos.

El número de viajes presenta una alta correlación positiva con el número de fatalidades (0.978), con el número de accidentes (0.991) y bajas correlaciones positivas con el número de vehículos y con el nivel de gestión de riesgo.

El nivel de gestión de riesgo tiene una alta correlación positiva con el número de vehículos (0.984), pero baja correlaciones bajas con las otras variables.

La demanda de combustible claro (gasolinas, diesel, kerosene, avgas, avjet) es la mayormente demandada de entre los derivados del petróleo. En la Tabla 2, se muestra el histórico de demanda de combustible claro desde el 2007 al 2016.

Tabla 2: Demanda de hidrocarburos claros 2007 al 2016 (Galones).

\begin{tabular}{|l|l|}
\hline Año & $\begin{array}{l}\text { Hidrocarburos Claro } \\
\text { (Galones })\end{array}$ \\
\hline 2007 & $514,933,375$ \\
\hline 2008 & $555,171,137$ \\
\hline 2009 & $593,322,845$ \\
\hline 2010 & $662,382,420$ \\
\hline 2011 & $749,809,576$ \\
\hline 2012 & $736,074,104$ \\
\hline 2013 & $797,083,316$ \\
\hline 2014 & $894,972,032$ \\
\hline 2015 & $864,020,648$ \\
\hline $2016 *$ & $896,558,871$ \\
\hline
\end{tabular}

Fuente: (Secretaría Nacional de Energía, 2020).

A partir de esta información, fue posible generar un modelo polinomial de series de tiempo, que se muestra en la Gráfica 3. Dicho modelo puede describirse como: 


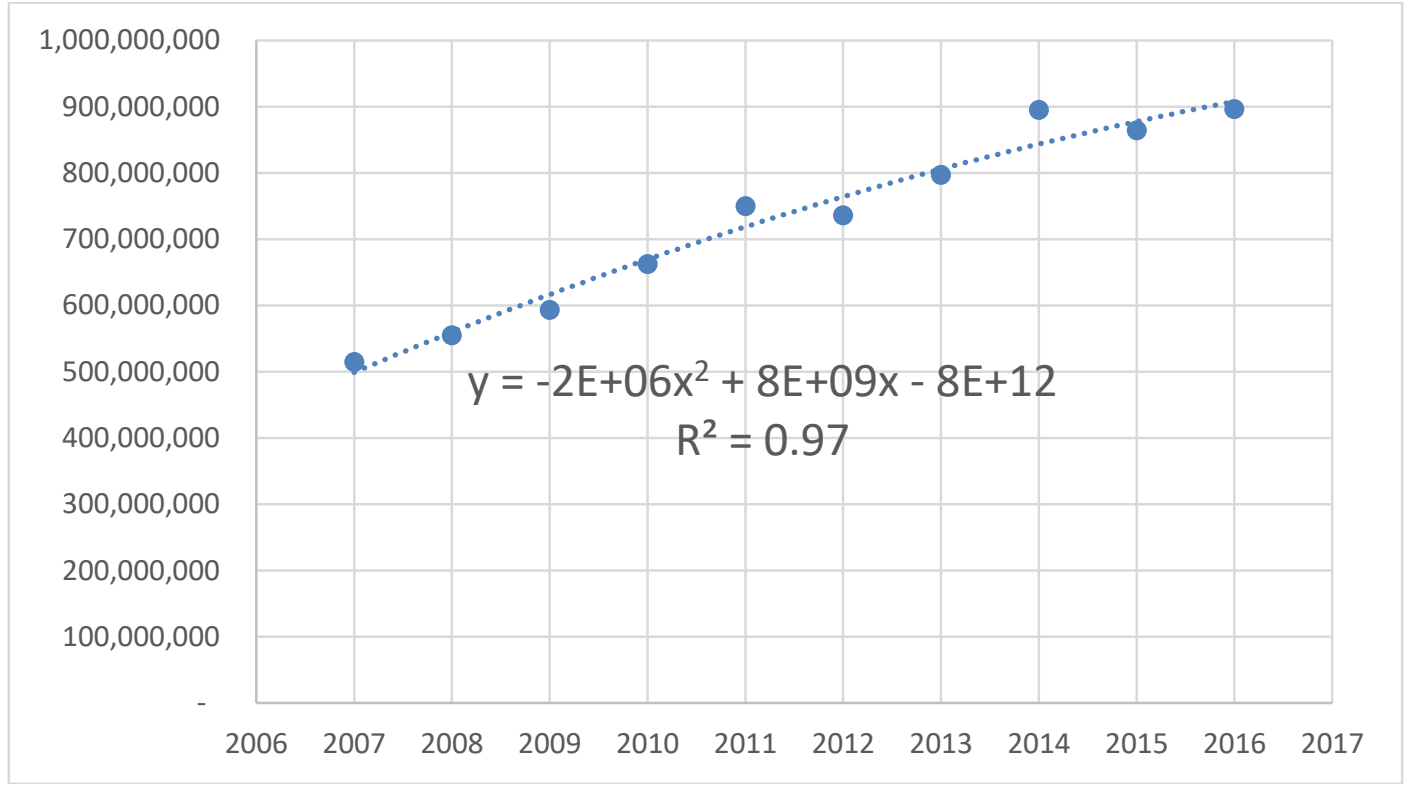

Gráfico 3: Gráfico de dispersión y de tendencia entre la Demanda y el tiempo (años).

Ecuación 1: Demanda de combustible claro = -1.90 x 106 (Año) $2+7.71 \times 109$ (Año) $-7.80 \times 1012$

El coeficiente de correlación resultante es de 0.98 a lo cual, es una excelente relación de ambas variables. La relación lógica, que debe existir, es que en la medida que exista demanda habrá más viajes a lo que se analiza su relación a continuación en la Gráfica 4 la cual se demuestra una relación polinomial de segundo grado.

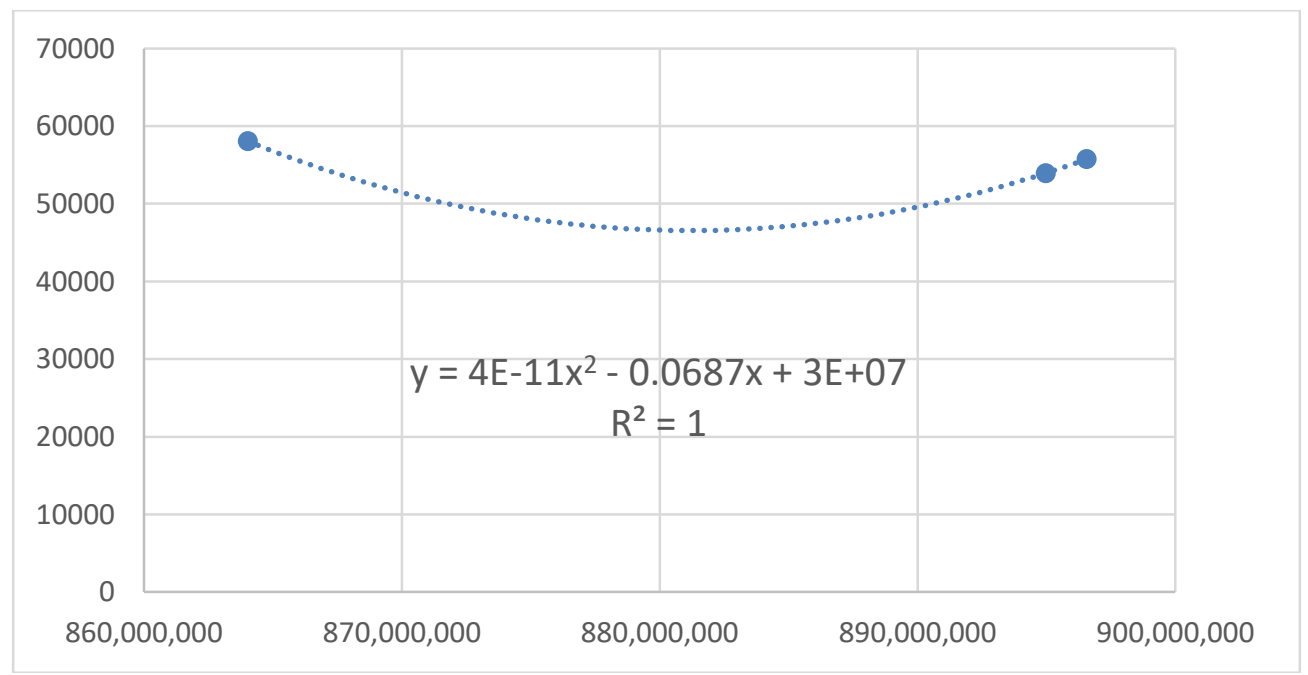

\section{Gráfico 4: Número de viajes en función de la Demanda.}

Ecuación 2: Número viajes $=3.90 \times 10-11($ Demanda) $2-0.07$ (Demanda) $+3.03 \times 107$ 
Bajo estos niveles de correlación, se puede utilizar los siguientes modelos para pronosticar lo siguiente: El número de accidentes en función de la cantidad de viajes. En la Gráfica 5 se muestra la línea de tendencia entre el número de accidentes en función de la cantidad de viajes.

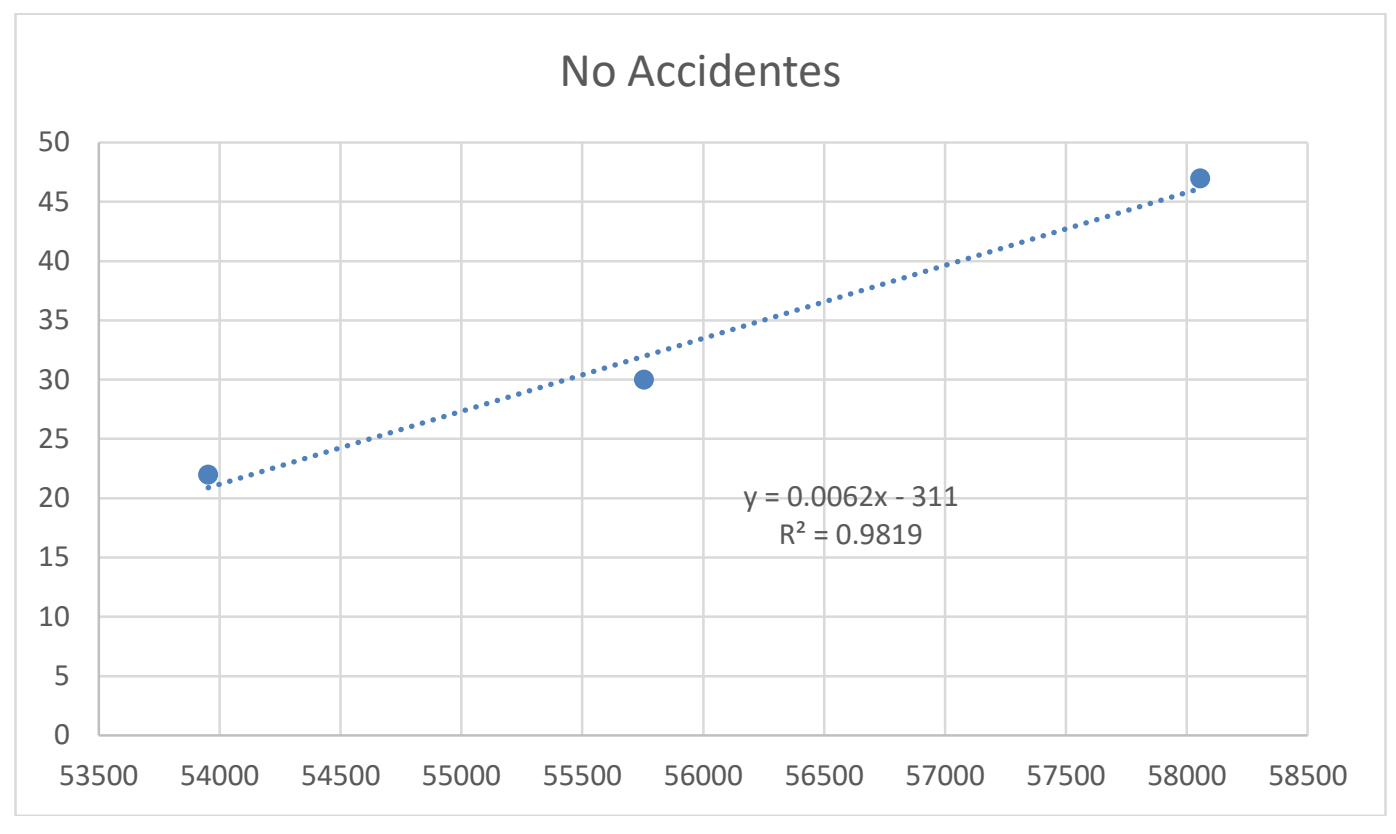

Gráfico 5: Tendencia del número de accidentes viales en función de la cantidad de viajes.

La ecuación de correlación sería la siguiente:

Ecuación 3: Número accidentes $=6.2 \times 10-3($ cantidad de viajes $)-311$

Sin embargo, el número de accidentes tiene una dependencia también del nivel de gestión de riesgo, para ello se procede a realizar el cálculo de la probabilidad de accidentabilidad por viaje dado un nivel de gestión de riesgo. Al realizarse el cálculo de la tasa de fatalidad por accidente ocurrido en el transporte de combustible claro, es posible, señalar que los administradores de transporte indicaron que tuvieron 99 accidentes de los cuales tuvieron 5 fatalidades lo que permite afirmar, que aproximadamente $5 \%$ de los accidentes ocurren fatalidades. Esto indica que la tasa de mortalidad por accidente en el transporte de combustible claro, es mucho mayor al promedio general del país al cual es de 1\% (volver a ver Tabla 2).

La probabilidad de accidentabilidad dado un nivel de gestión de riesgo (a) es determinado de la siguiente forma: se obtienen los totales para los tres años medidos 2014, 2015 y 2016, en las siguientes variables:

Número de viajes (NV)

Número de accidentes (A)

Nivel de gestión de riesgo (NGR)- 
Luego se agrupan por Niveles de Gestión de Riesgos (NGR), en intervalos de tamaño 20\%, se determina la probabilidad de accidentabilidad, dado el nivel de gestión de riesgo (a) dividiendo el número de accidentes (A) y el número de viajes realizados (NV).

Dentro de los niveles de gestión de riesgo (NGR), entre $20 \%$ a $40 \%$ se tuvo un total acumulado de 2,396 viajes de los cuales se dieron 2 accidentes por lo que la probabilidad de accidentabilidad de las compañías, que se encuentran en niveles de gestión de riesgo entre $20 \%$ a $40 \%$ es de 0.00083 . La tabla completa con los cálculos respectivos por Nivel de gestión de riesgo (NGR) y sus respectivas cantidades de viajes y accidentes se muestran en la Tabla 3.

Tabla 3: Cálculo de la probabilidad de accidentes dado un determinado Nivel de Gestión de Riesgo Nivel de GR (NGR) - Suma de Número de Viajes (NV) - Suma de Número de Accidentes (A) Probabilidad de Accidentes por Viaje (a)

\begin{tabular}{|l|l|l|l|}
\hline Nivel de GR (NGR) & $\begin{array}{l}\text { Suma de } \\
\text { Número de } \\
\text { Viajes (NV) }\end{array}$ & $\begin{array}{l}\text { Suma de Número } \\
\text { de Accidentes (A) }\end{array}$ & $\begin{array}{l}\text { Probabilidad de } \\
\text { Accidentes por } \\
\text { Viaje (a) }\end{array}$ \\
\hline $20 \%-40 \%$ & 2,396 & 2 & 0.00083 \\
\hline $41 \%-60 \%$ & 4,395 & 3 & 0.00068 \\
\hline $61 \%-80 \%$ & 6,435 & 4 & 0.00062 \\
\hline $80 \%-100 \%$ & 154,536 & 90 & 0.00058 \\
\hline
\end{tabular}

Con estos resultados de probabilidades, se demuestra que la hipótesis de que a mayores niveles de gestión de riesgo (NGR) menor probabilidad de accidentabilidad.

Diagrama de influencias para el modelo de simulación.

El diagrama de influencias mostradas en la Gráfica 6, se muestra el diagrama de influencias que resumen las relaciones de las variables consideradas en el modelo de simulación para determinar el número de fatalidades por accidentes de tránsito, en las compañías de transporte evaluadas. 


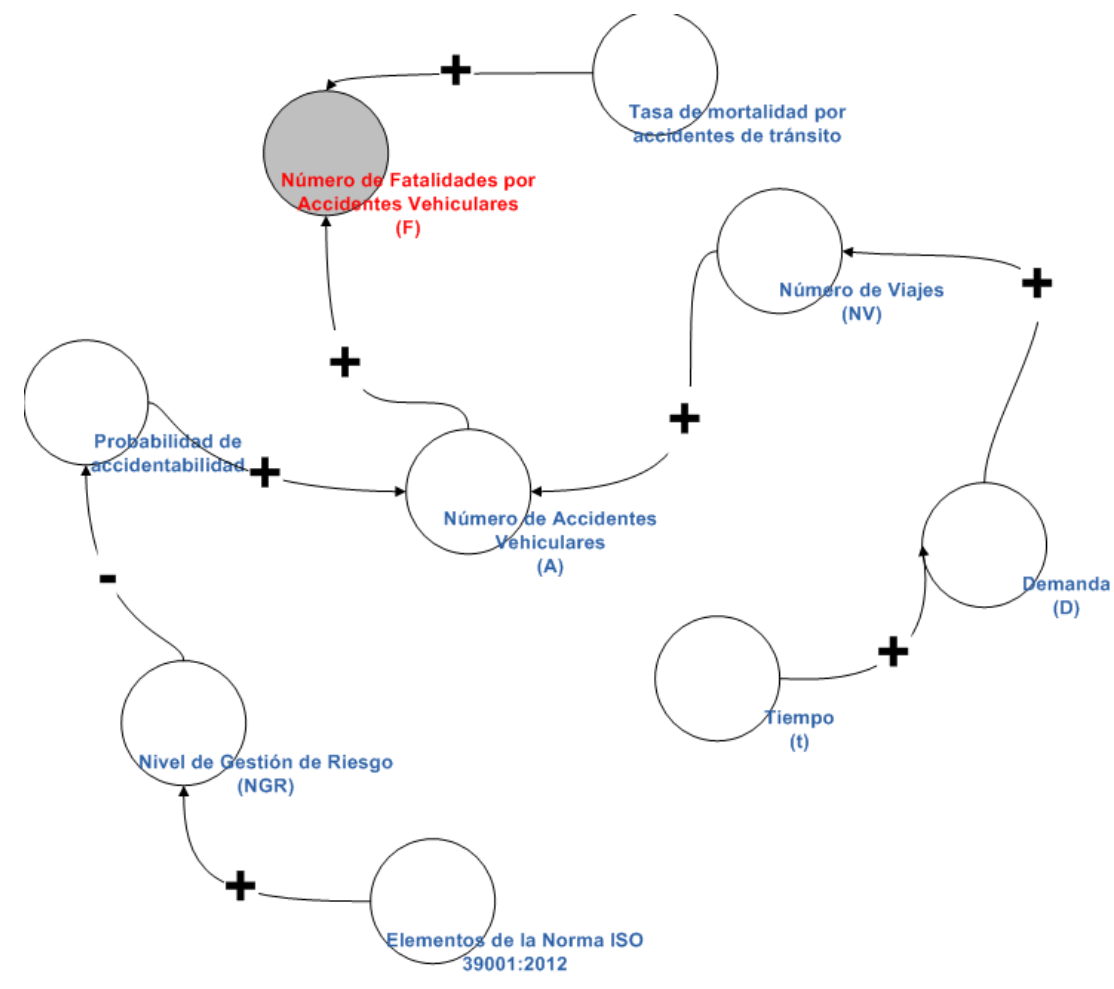

Gráfico 6: Diagrama de influencias para el modelaje del número de accidentes y fatalidades viales..

\section{Modelo utilizando Vensim ${ }^{\circledR}$.}

Con el diagrama de influencias, se procedió a confeccionar el diagrama de Forrester el cual, permite observar las relaciones de las variables según sean variables de nivel o variables de flujo.

Para el diagrama de Forrester, se tomó que las variables de nivel serían la cantidad de fatalidades (F), cantidad de accidentes (A), número de viajes (NV) ocurridas en el lapso 2017 al 2021; las variables de flujo serían el tiempo (t), la demanda (D), Nivel de Gestión de riesgo (NGR) y esta a su vez tiene dependencias de los puntajes alcanzados en cada uno de los elementos de la norma ISO 39001:2012, en los cuales cada uno de ellas representa a una variable. Se ha utilizado una variable de control llamada, "Tasa de incremento" para hacer los escenarios de aumento o no del nivel alcanzado en cada elemento de la norma. Adicionalmente, se han colocado en el modelo dos tasas las cuales son: probabilidad de accidentabilidad y la tasa de mortalidad por viaje.

El período de evaluación configurado fue de 5 años (2017 al 2021). El modelo final fue desarrollado con el uso del software Vensim ${ }^{\circledR}$ la cual se muestra el diagrama de Forrester configurado. En la Gráfica 7 se muestra el diagrama de Forrester (UNAD, 1995) hecho en Vensim ${ }^{\circledR}$. 


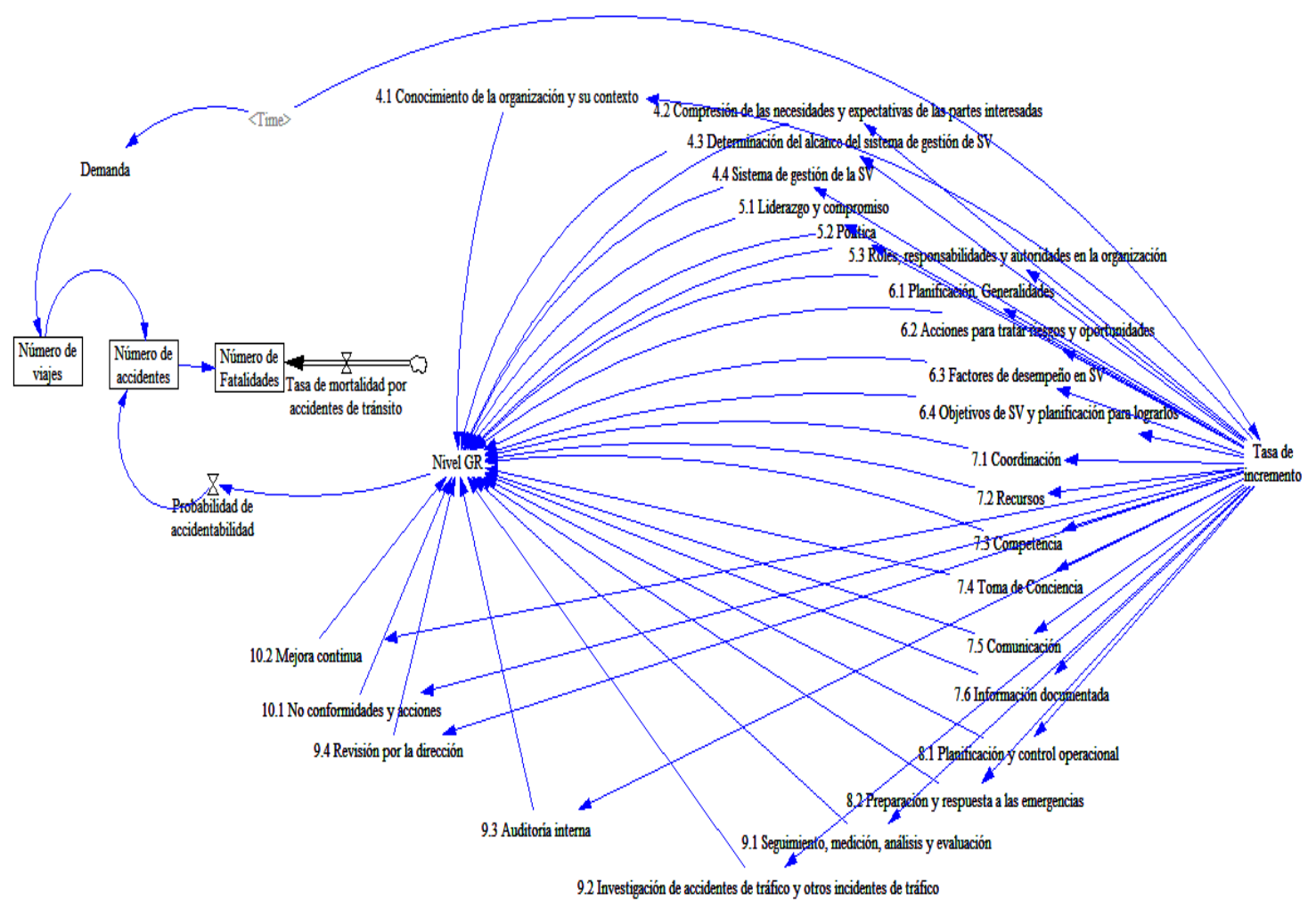

Gráfico 7: Modelo de simulación utilizando Vensim ${ }^{\circledR}$.

Escenarios evaluados con Vensim ${ }^{\circledR}$..

Para la configuración de las variables se debe tomar en cuenta, que se simularán dos escenarios:

Escenario 1: se tiene una tasa de crecimiento continuo de $10 \%$ anual en cada uno de los elementos de la norma ISO 39001:2012, teniendo como valor inicial cada elemento de la norma mencionada en 20\% y como valor máximo 100\%

Escenario 2: se mantiene constante el valor de cada elemento de la norma ISO 39001:2012 en un valor de $20 \%$ durante los cinco años de simulación.

Para determinar la probabilidad de fatalidad dado un accidente de tránsito en el transporte de combustible claro, se determinó que en 97 accidentes tenidos en el periodo del 2014 al 2016, se tuvieron 5 fatalidades. Dado ello, la probabilidad de fatalidad dado un accidente de tránsito es de $5 \%$ en el transporte de combustible claro.

\section{Resultados de la simulación Vensim®..}

En la Gráfica 8 se muestra el pronóstico de demanda de combustible para los años de 2017 hasta el año 2021: 


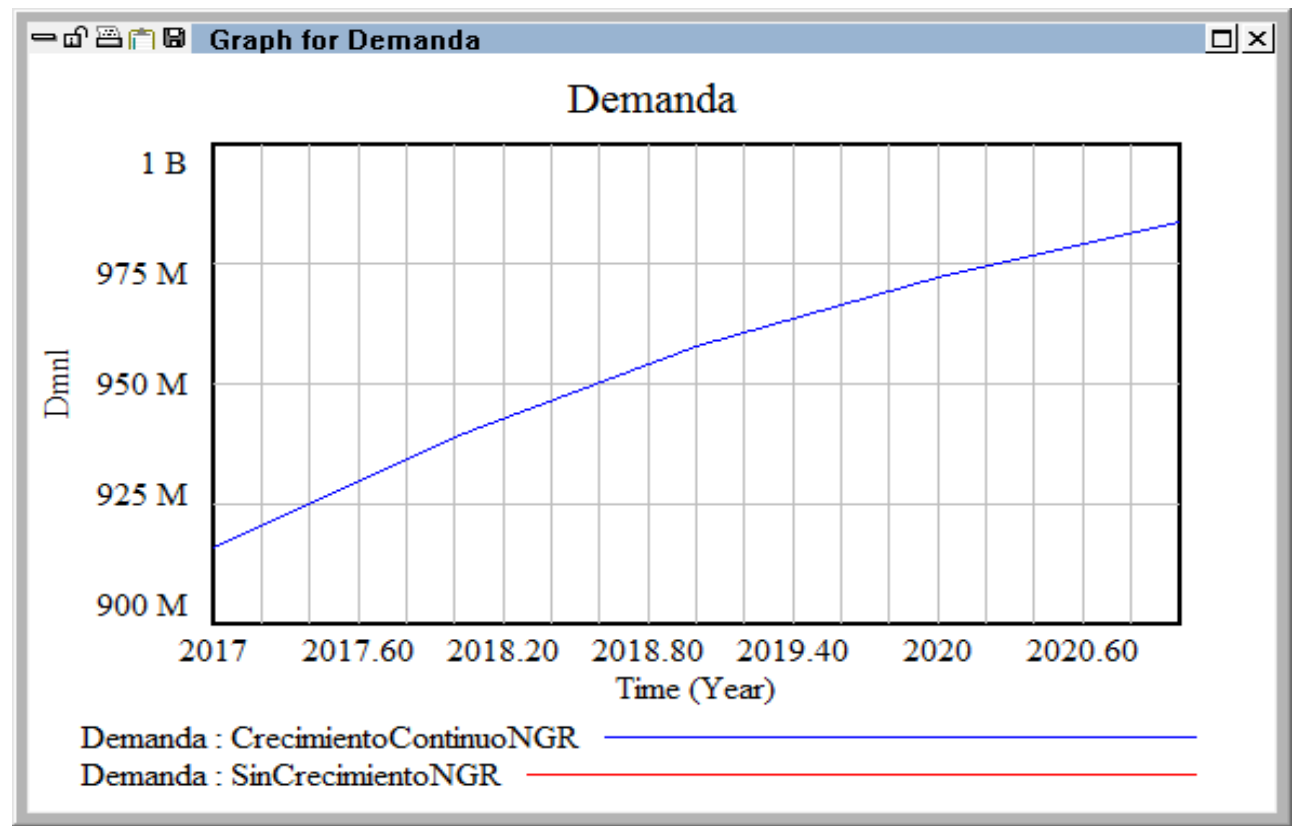

Gráfico 8: Pronóstico de la demanda de combustible claro para el periodo 2017 al 2020.

La siguiente variable para determinar, es el número de viajes esperado $\mathrm{E}(\mathrm{NV})$ para el periodo del 2017 al 2021. Esta variable, debe tener el mismo comportamiento para ambos escenarios (con y sin aumento en el NGR). La demanda esperada E(D) de combustible claro es la variable correlacional utilizada para determinar los números de viajes esperados $\mathrm{E}(\mathrm{NV})$.

En cuanto a los accidentes esperados, en base a la cantidad de viajes E(NV) y la probabilidad de accidentabilidad, dado el nivel de gestión de riesgo (a) el cual es determinado por el producto de ambas, según sea el Nivel de Gestión de Riesgos (NGR). E(A) = E(NV) * P(a/NGR)

Donde:

$\mathrm{E}(\mathrm{A})=$ el número esperado de accidentes.

$\mathrm{E}(\mathrm{NV})=$ Número esperado de viajes

$\mathrm{P}(\mathrm{a} / \mathrm{NGR})=$ la probabilidad de accidentabilidad dado un nivel de gestión de riesgo (NGR)

Volviendo a recalcar, que los dos escenarios utilizados para el periodo de cinco años (2017 al 2021) son los siguientes:

Escenario 1: cada elemento de la norma ISO 39001:2012 inicia en un nivel de 20\% y luego se dan aumentos de 10\% anual durante la simulación.

Escenario 2: se asume que todos los elementos de la norma se mantienen en una constante de 20\% durante los cinco años.

A continuación, se comparan en la Gráfica 9 los dos escenarios esperados teniendo en color rojo el Número de fatalidades esperadas E(F) sin incremento del NGR y con línea de color azul, se muestra el comportamiento de la cantidad de fatalidades esperadas $\mathrm{E}(\mathrm{F})$ considerando un aumento progresivo de $10 \%$ en el NGR. 


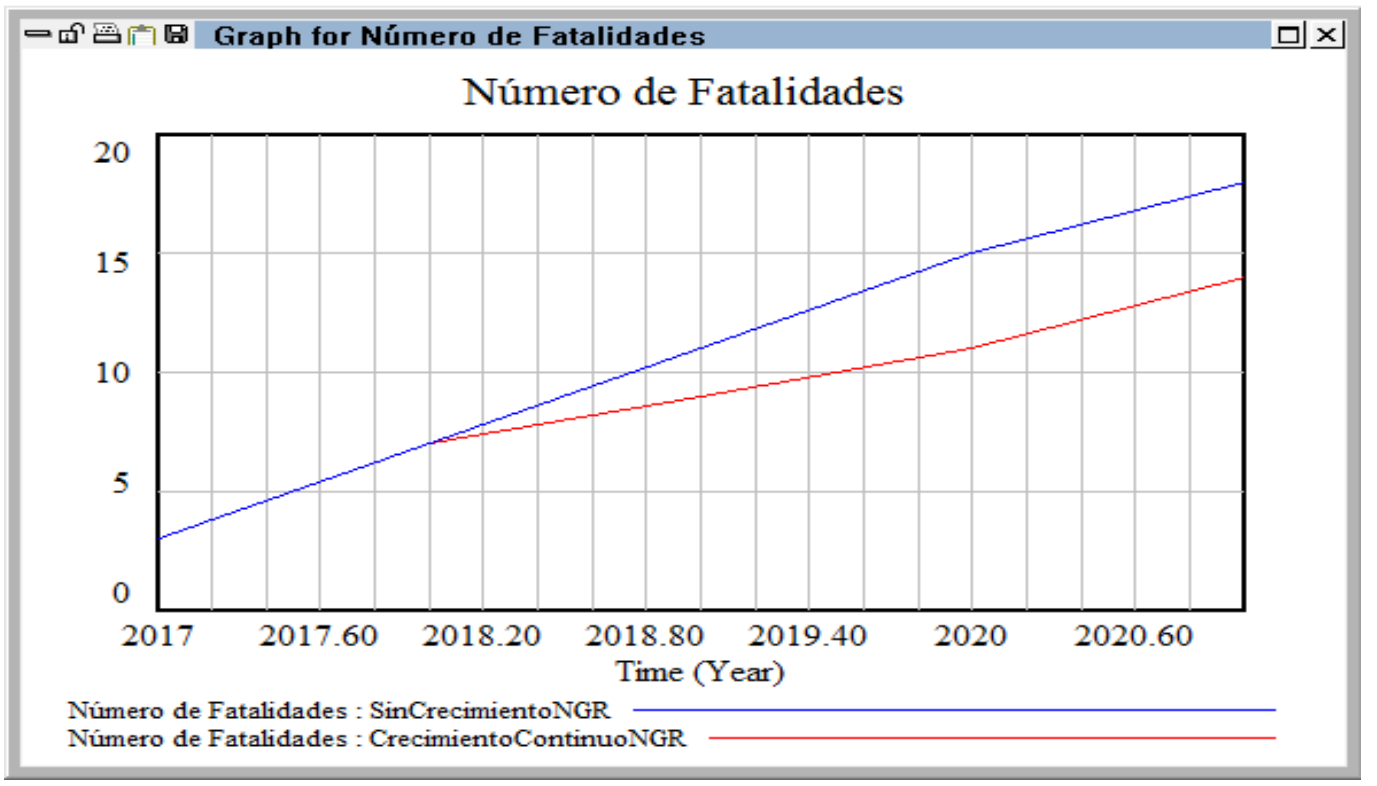

Gráfico 9: Los dos escenarios de cantidad de fatalidades dados según el nivel de gestión de riesgo para el periodo 2017 a 2021. Con un 10\% de incremento en gestión de riesgo.

Lo que se muestra de los resultados de la simulación en cuanto a las fatalidades esperadas $\mathrm{E}(\mathrm{F})$ es que si se mantiene un nivel de Gestión de Riesgo constante (20\%), la cantidad de fatalidades esperadas $\mathrm{E}(\mathrm{F})$, es mayor que si el nivel de gestión de riesgo aumentase con los años.

En la Tabla 4 se hace una comparación de los dos escenarios (con y sin crecimiento del NGR) notándose que la cantidad de fatalidades esperadas E(F), bajan cuando el NGR aumenta; se puede tomar como ejemplo el resultado del año 2020, en el que el NGR de 60\% llega a reducir las fatalidades esperadas $\mathrm{E}(\mathrm{F})$ hasta un $27 \%$ comparado con el escenario de no aumentar el NGR.

Tabla 4: Comparación de los escenarios con y sin crecimiento del NGR en cuanto al número de fatalidades.

\begin{tabular}{|c|c|c|c|c|c|}
\hline Año & NGR & $\begin{array}{c}\text { Fatalidades } \\
\text { esperadas }\end{array}$ & NGR & $\begin{array}{c}\text { Fatalidades } \\
\text { esperadas }\end{array}$ & Dif\% (F) \\
\hline $\mathbf{2 0 1 7}$ & $\mathbf{2 0 \%}$ & 3 & $30 \%$ & 3 & $\mathbf{0} \%$ \\
\hline $\mathbf{2 0 1 8}$ & $\mathbf{2 0 \%}$ & 7 & $40 \%$ & 7 & $\mathbf{0} \%$ \\
\hline $\mathbf{2 0 1 9}$ & $20 \%$ & 11 & $50 \%$ & 9 & $-18 \%$ \\
\hline $\mathbf{2 0 2 0}$ & $20 \%$ & 15 & $60 \%$ & 11 & $-\mathbf{2 7} \%$ \\
\hline $\mathbf{2 0 2 1}$ & $20 \%$ & 18 & $70 \%$ & 14 & $-\mathbf{2 2} \%$ \\
\hline
\end{tabular}


Este resultado hace concluir que en la medida que el nivel de gestión de riesgo aumenta, la cantidad de fatalidades esperadas disminuyen y esto apoya la hipótesis de este trabajo investigativo que indica que en la gestión del riesgo es un factor determinante en la reducción de fatalidades por accidentes de tránsito.

En cuanto a los accidentes de tránsito esperados $\mathrm{E}(\mathrm{A})$ de estos transportistas de combustible claro para el periodo 2017 al 2021 se muestran los resultados en la Gráfica 10.

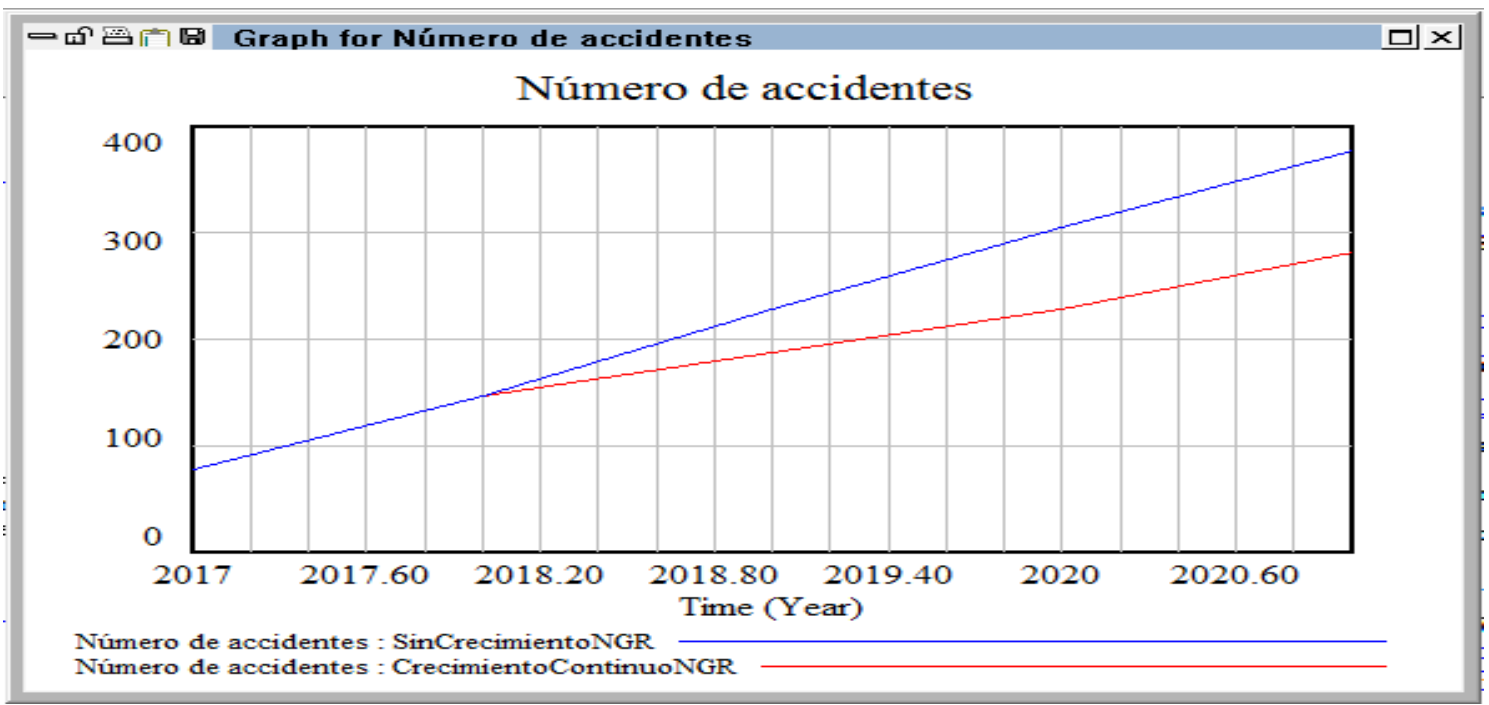

Gráfico 10: Los dos escenarios de cantidad de accidentes dados según el nivel de gestión de riesgo para el periodo 2017 a 2021. Con un 10\% de incremento en gestión de riesgo.

Al igual que en la cantidad de fatalidades esperadas $\mathrm{E}(\mathrm{F})$, también se observa una reducción del número de accidentes $\mathrm{E}(\mathrm{A})$, cuando el nivel de gestión de riesgo aumenta llegando hasta una reducción de la cantidad de accidentes en un 25\%. Los cálculos se muestran en la Tabla 5.

Tabla 5: Comparación de los escenarios sin y con crecimiento del NGR en cuanto al número de accidentes esperados.

\begin{tabular}{|l|l|l|l|l|l|}
\hline Año & NGR & $\begin{array}{l}\text { Accidentes } \\
\text { esperados }\end{array}$ & NGR & $\begin{array}{l}\text { Accidentes } \\
\text { esperados }\end{array}$ & Dif\% (F) \\
\hline 2017 & $20 \%$ & 77 & $30 \%$ & 77 & $0 \%$ \\
\hline 2018 & $20 \%$ & 146 & $40 \%$ & 146 & $0 \%$ \\
\hline 2019 & $20 \%$ & 228 & $50 \%$ & 187 & $-18.0 \%$ \\
\hline 2020 & $20 \%$ & 305 & $60 \%$ & 228 & $-25.2 \%$ \\
\hline 2021 & $20 \%$ & 377 & $70 \%$ & 282 & $-25.2 \%$ \\
\hline
\end{tabular}

A pesar de que la cantidad de viajes esperados $\mathrm{E}(\mathrm{NV})$ va en aumento, así también el número de accidentes, pero a un nivel de crecimiento mucho menor que la cantidad de viajes generados a mayores niveles de gestión de riesgo. 


\section{Discusión}

Las conclusiones a las que se ha llegado, luego de este trabajo investigativo se pueden resumir en los siguientes puntos:

En las compañías de transporte, la gestión de riesgo operacional se resume en la gestión de riesgo vial, por lo que la gestión de la seguridad vial es la equivalencia a la gestión del riesgo en las operaciones de compañías de transporte.

El sector de transporte de combustibles claro en Panamá ha ido evolucionando en su implantación de niveles de gestión de riesgo hasta llegar a contar con muy buenos niveles en gestión de seguridad vial.

La demanda de combustible claro esperada es sumamente alta por lo que hay una expectativa que aumente significativamente la cantidad de viajes del transporte de combustible claro. Esto, hace aumentar la exposición de los equipos y que se incremente el número de compañías pequeñas que se sumen al transporte y por consecuencia aumente el riesgo de accidentes y fatalidades.

Los accidentes y fatalidades por accidentes de tránsito tienen varias variables que las generan. La gestión de riesgo en seguridad vial es un factor determinante para reducirlas, pero no la única.

\section{Recomendaciones}

Ahora se hace la presentación de las recomendaciones, que se puede ofrecer como resultado de esta investigación:

Se debe aplicar auditorías por parte de una autoridad, tal vez del Estado, para que se tenga una medida del nivel de gestión de riesgo con muestras de evidencias del cumplimiento en lugar de solo la aplicación de la encuesta.

La herramienta presentada, en este trabajo investigativo puede ser utilizada en futuras auditorías con modificaciones en las columnas dicotómicas anuales para ser evaluaciones del periodo elegido, pero bajo una escala Likert.

La industria del transporte de combustible claro necesita determinar y documentar en sus sistemas de gestión de la seguridad vial como resultados esperados la disminución de accidentes y por ende las fatalidades generadas en las operaciones.

Se deben establecer sistemas de auditorías a los sistemas de gestión de riesgo de las compañías de transporte, principalmente para las empresas pequeñas (de tres o menos equipos). Estas compañías pueden ser apoyadas por las empresas que las sub contratan para transportarles productos, ya que muchas veces sus clientes poseen los recursos y conocimientos para mejorar sus procesos.

Las autoridades, deben de implementar programas de ayuda a las compañías chicas del transporte para que mejoren sus sistemas de gestión de seguridad vial. Estas, pueden ser por medio de capacitaciones en seguridad vial por medio de INADEH y UDELAS, por ejemplo.

Se propone investigar, el impacto de la fatiga en los accidentes de tránsito de los camiones de carga ya que es el tercer punto más mencionado como razón de los accidentes viales y que en Panamá no hay una legislación propiamente que regule los tiempos de jornada de los conductores del transporte de carga.

Panamá debe considerar la adopción de normas internacionales de gestión de riesgo como la ISO 39001:2012. Con estas normas se pueden realizar capacitaciones y auditorías a los transportistas para poder dar guías y correcciones que salven vidas. 


\section{Bibliografía}

International Standard Organization. 2017. ISO 39001:2012. Road traffic safety (RTS) management systems - Requirements with guidance for use. [En línea] 2017. [Citado el: 01 de marzo de 2017.] https://www.iso.org/standard/44958.html.

Organización Mundial de La Salud. 2016. Decenio de Acción para la Seguridad Vial 2011-2020. [En línea] 1 de agosto de 2016. http://www.who.int/roadsafety/decade_of_action/es/.

Sáenz, Estefanía y Moya, Marian. 2017. Evaluación del nivel de gestión de la seguridad vial basado en las buenas prácticas para el transporte de carga. Panamá : Universidad Tecnológica de Panamá, 2017.

Secretaría Nacional de Energía. 2020. Información sobre energía en Panamá. [En línea] 2020. [Citado el: 10 de enero de 2020.] http://www.energia.gob.pa/.

Sminkey, Laura. 2011. Decenio de acción para la seguridad vial 2011-2020. Geneva: World Health Organization, 2011.

-. 2010. Decenio de Acción para la Seguridad Vial 2011-2020. Geneva : World Health Organization, 2010.

Sterman, John. 2000. Business Dynamics: Systems Thinking and Modeling for a Complex World. s.l.: Irwin/McGraw-Hill, 2000.

Torres, Práxedes. 2017. La gestión de riesgo como factor determinante en la reducción de fatalidades por accidentes de tránsito en el transporte. Panamá : Universidad del Istmo, 2017.

- 2017. La gestión de riesgo como factor determinante en la reducción de fatalidades por accidentes de tránsito en el transporte. Panamá : Universidad del Istmo, 2017.

UNAD. 1995. Lección 17: Diagramas de Forrester. [En línea] 1995. [Citado el: 1 de Febrero de 2016.]

http://datateca.unad.edu.co/contenidos/301126/301126/leccin_17_diagramas_de_forrester.html. 